\title{
Peptides as Drug Candidates: Limitations and Recent Development Perspectives
}

\author{
Yusuf A. Haggag*1, Ahmed A. Donia ${ }^{1,2}$, Mohamed A. Osman' ${ }^{1}$, Sanaa A. El-Gizawy ${ }^{1}$ \\ ${ }^{1}$ Department of Pharmaceutical Technology, Faculty of Pharmacy, Tanta University, Tanta, Egypt \\ ${ }^{2}$ Department of Pharmaceutical Technology, Faculty of Pharmacy, Menofia University, Menofia, Egypt
}

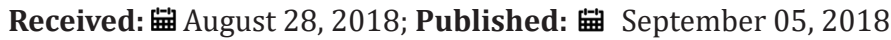

*Corresponding author: Yusuf A Haggag, Department of Pharmaceutical Technology, Faculty of Pharmacy, Tanta University, Egypt

Abbreviations: GLP-1: Glucagon-Like Peptide-1; PEG: Polyethylene Glycol; Gamma IgG: Immunoglobulin; FcRn: Fc Receptor

\section{Introduction}

Peptides can be defined as polypeptide chains of 50 or less amino acids or 5000 Da in molecular weight characterized by a high degree of secondary structure and lack of tertiary structure. Therapeutic peptides have traditionally been derived from nature as naturally occurring peptide hormones (known as bioactive peptides), genetic/recombinant libraries and chemical libraries [1]. The recent technologies used for peptides production include chemical synthesis, enzymatic synthesis, recombinant DNA biotechnology, cell-free expression and transgenic animal or plant species. The possibility to determine the most suitable one for each peptide production depends on peptide size. The use of unnatural amino acids and pseudo-peptide bonds make chemical synthesis offering wider chemical diversity than other peptide derivatives produced by recombinant DNA technologies or bio-catalysis. The advantage of Large-scale production of chemical synthesis makes it a viable technology especially for the production of small and medium-sized peptides ranging from approximately 5 to 50 residues [2].

Over the years, peptides have been evolved as promising therapeutic agents in the treatment of different disease as cancer, diabetes, and cardiovascular diseases. Therapeutic application of peptides for other treatments is growing rapidly [3]. Bioactive peptides like glucagon-like peptide-1(GLP-1) used for the treatment of diabetes, gastrin-releasing peptide used in cancer treatments, ghrelin peptide to treat obesity, and defensin, which used as an antimicrobial agent have several intrinsic advantages over peptides derived from other random peptide libraries because of enhanced in vivo stability and their ability to activate receptors or disrupt protein-protein interactions better than other peptides
[4]. Discovery of several tumor-related peptides and proteins also protein/peptide receptors is supposed to create a new revolution wave of more promising, effective and selective anticancer drugs in the future. Therapeutic anticancer peptides will capture the largest share of the cancer therapeutic market [2]. This mode of cancer treatment including peptides, proteins and monoclonal antibodies is termed "biologics" treatment option [5].

About $75 \%$ from the whole peptide drugs in the market that gained total global sales over $\$ 1$ billion are used directly in cancer treatment like (leuprolide, goserelin, and octreotide peptides). There are several hundred peptide candidates under clinical trials development. Approximately, 18\% from more than 2000 peptides entering clinical studies were most frequently indicated for cancer [6,7]. Peptides as potential therapeutic drugs offer many advantages over protein and antibodies. Peptides have the advantage of better tissue penetration because of its smaller sizes and less immunogenicity than recombinant antibodies and proteins at the biological level [8] also at the cost level, peptides are produced with lower manufacturing costs (synthetic technology versus recombinant one) with more activity per unit mass [9]. Peptides are superior to antibodies at the physical level in terms of accepted room temperature storage. Furthermore, over small organic molecules due to its higher targets affinity, specificity and efficacy also lower toxicity effects because of their safe metabolites [4].

\section{Limitations to the Use of Peptides as Drug Candidates}

Peptides as a type of bio drugs have several drawbacks that hinder their therapeutic application. Their undesirable 
physicochemical properties, such as variable solubility, low bioavailability and limited stability make their systemic delivery difficult [10]. The bottlenecks of peptide and protein drug delivery are generally attributed to the following points:

a. Low oral bioavailability and shorter half-life. Peptide hydrophilicity is responsible for its poor permeability through physiological barriers and biological membranes. In addition to, their limited stability due to rapid digestion by protolytic enzymes of the digestive system and blood plasma as well as rapid clearance from the circulation within few minutes by the liver and kidneys [11]. Therefore, parenteral administration with repeated doses is generally required that might result in an oscillating concentration of the peptide in the blood [12].

b. Poor specific bio-distribution due to high conformational flexibility, resulting sometimes in a lack of receptor selectivity and activation of different target receptors leading to side effects [11].

c. Eventual risk of immunogenic effects as most peptide and protein drugs appear immunogenic [13].

d. High production costs of synthetic peptides than small molecules of the same molecular weight of 5000D by more than 10-fold [14].

e. Therapeutic peptides and proteins also tend to undergo denaturation, aggregation, and adsorption which limit their active concentration and proper function in vivo [15].

\section{Improving In vitro and In vivo Stability of Peptides}

The low bioavailability and short half-life of peptides are the major hurdles for peptide therapeutics application due to high degradation by gastrointestinal, plasma and tissue peptidases also their rapid clearance from the circulation within minutes to hours after administration. Consequently, there is no sufficient exposure to the target tissue to produce an in vivo effect which finally limits their therapeutic use. To address these issues, numerous acceptable technologies have been developed to increase plasma residence time of peptides in vivo [2,4]. Before the enhancement of the bioavailability and serum half-life in vivo, most peptides must be altered in vitro to increase its protease resistance by reduction of degradation by tissue and serum proteases and peptidases. This entails In vitro chemical alteration and modification, by different methods that include $\mathrm{C}$-terminal amidation, $\mathrm{N}$ terminal acetylation and use of non-natural amino acids at different labile sites within a peptide. Chemical modifications by cyclization via disulfide bonds to decrease the high conformational flexibility of linear peptides substantially increased proteolytic stability [16].

The increase in peptide stability by chemical modifications is clear when peptides assembled totally or partly from D-amino acids instead of natural L-amino acids. Incorporation of D-amino acids showed high peptide stability due to less susceptibility to proteolytic degradation also in some cases increased its binding affinity [17]. Non-natural amino acids or phosphorylated modified amino acids can be directly incorporated, however, after every chemical modification; peptides need to be retested for their binding affinity. Chemical modification methods might alter the lead peptide efficacy so that it needs further alteration to restore the biological activity. This was nicely shown for the peptide interfering with binding of Mdm-2 to p53 [18]. A recent patent method using the in vitro CIS display system can combine both protease resistance and selection for biological activity by using L-amino acid peptides with built-in protease resistance, combined with high affinity binding activity to enhance and maintain in vitro serum activity of a biologically active tested peptide from a few minutes to nearly $24 \mathrm{~h}$ [19].

Other approaches to increase in vitro stability of the peptides can be achieved by addition of different stabilizing agents acting through different mechanisms. The thermal stability of peptides and proteins can be improved by addition of sugars (sucrose, maltose, trehalose or glucose) or salts (potassium phosphate, ammonium sulphate or sodium citrate), as well as heparin in order to achieve self-association and solubility modulation [20]. Chelating agents, such as EDTA, form complexes with metal dependent proteases/ peptidases, and thus inhibit catalytic degradation processes [21]. Cyclodextrins, especially hydroxy-propyl-cyclodextrins, can be used to stabilize peptides and proteins; however the mechanism of action is not clear. Moreover, non-ionic surfactants as (Pluronic F68) can stabilize peptides and proteins against self-aggregation. On the other hand, anionic (SDS) and cationic (cetrimide) surfactants facilitate peptides and proteins absorption across biological membranes [22]. In vivo stability of peptide and protein drugs in the bloodstream, where they are exposed to proteolytic degradation can be achieved by different approaches to enhance serum residence times for peptides. Polyethylene glycol (PEG) has many advantages that will be transferred to the peptides such as high-water solubility, free mobility in solution and low immunogenicity that make it an ideal carrier for peptides.

PEG is often attached to the $\mathrm{N}$ or $\mathrm{C}$ terminal of peptides to form PEG-peptide conjugates serve to increase the overall size, the hydrodynamic radius and increase the molecular weight $(>50$ $\mathrm{kDa}$ ) of peptide conjugates in order to not to be filtered out by the kidneys, also protect peptides from degradation by exopeptidases and therefore improve their in vivo stability [16]. Although, PEG modification can inhibit peptide function, it significantly decreases its immunogenicity so that testing of several different PEG derivatives with different molecular weights is still continuous. Conjugation of (PEG) to proteins such as Interferon-a 2a (Pegasys $®$, Roche) or Interferon-a 2b (PEG-Intron, Schering), is now a widely approved method to increase serum residence times [23]. Another method used to increase peptide molecular size is the genetic fusion of peptides to the natural antibody constant region Fc domain of 
human gamma immunoglobulin (IgG) offering an added advantage of the IgG protection function of the neonatal Fc receptor (FcRn).

A major disadvantage is the possibility of steric hindrance if the fused peptide is large which may decrease the drug potency. The 'peptibody' AMG 531 (developed by Amgen) beside three other proteins fused to Fc have been approved as therapeutics: Enbrel $₫$, Amevive $\AA$ and Orencia $\AA$ [24]. Alternative approach is to fuse peptides to albumin by chemical conjugation with covalent linkage to the free cysteine at position $34^{\text {th }}$ amino acid of albumin which benefits from half-life extension also effective in extending therapeutic peptide half-life by escaping renal clearance. Human Genome Sciences developed Albuferon, a type of interferon $\alpha$ peptide fused to albumin at the $C$ terminal, which is effective in prolonging drug half-life while maintaining drug potency [25]. Increasing peptides intracellular stability was done by associating or fusing a small peptide to a stabilizing protein (small ubiquitinrelated modifier SUMO-1) which is small, abundant and of human origin. Application of small amounts of peptides fused to SUMO-1 is safer and more effective as it did not interfere with the cellular function of this protein also worked well without cytotoxic effects so that, proteins can be used as a stabilizing domain for peptides but SUMO-1 was the first reported [26].

Similar approaches could be applied to stabilize peptide candidates using scaffolds by inserting peptides with both ends in a platform called scaffold protein in order to shift the equilibrium between a compact folded state and open unfolded state of the peptide toward the folded state the same as attaching them to a support protein (like SUMO-1) but the difference in SUMO-1 is that it is from one side. Scaffolds not only increase stability but also improve binding affinities by suppressing their flexibility in solution (entropy) before binding [9]. Scaffolds that can be used in cancer therapy to increase the stability of the peptides should have low immunogenicity, high affinity and no regions susceptible to proteolysis [6]. Meanwhile, multiple scaffolds have been described, such as fibronectin, lipocalin or ankyrin repeats [27]. The number of scaffolds is still growing and it is very likely that scaffolds which meet the criterira mentioned above will be developed as soon as possible in the future. The easy way to increase peptide and protein stability without modification or alteration of the peptide structure is by co-administration of protease inhibitors, such as sodium glycocholate, bacitracin reduces significantly degradation of insulin, vasopressin and calcitonin, and improve their essential absorption profile [28].

The recent promising strategy to improve the stability of therapeutic peptides and proteins is their encapsulation into a micro- or nanoparticle, in order to protect these labile drugs from the harsh environment in the body [29]. Nanoparticles and microspheres have been sought, not only as a means of protecting therapeutic peptides from degradation and prolonging their halflives in vivo, but also because they exhibit excellent controlled release properties and act as immunological adjuvants for protein antigens [30,31]. Controlled release formulations offers numerous advantages, including protecting therapeutic peptides and proteins over an extended period from degradation or elimination and increased patient compliance, also some modified nanoparticles have additional advantage of being able to specifically target bio drugs to diseased tissues, thereby reducing systemic exposure $[32,33]$. For biomedical applications, a number of different developed nanosystems have been used including liposomes, polymeric nanosystems, dendrimers, carbon nanotubes, crystals, fullerenes, and metal or inorganic nanoparticles.

\section{Conclusion}

Stability, biological efficacy, pharmacokinetic profile and immunogenicity are the most critical parameters to develop a peptide as a therapeutic agent. In recent years, some pharmaceutical companies contributed to rapidly growing interest in peptides as potential drug candidates. Peptides are generally supposed to be poor drug candidates because of their higher propensity to be rapidly metabolized also low oral bioavailability. The concept that these drugs are not orally available is always driving these companies to develop new diverse production strategies. These strategies aimed to reduce metabolism of these peptides together with developing different alternative routes of administration in order to increase the number of peptide-based drugs in the market.

\section{References}

1. Watt PM (2006) Screening for peptide drugs from the natural repertoire of biodiverse protein folds. Nature biotechnology 24: 177-183.

2. Vlieghe P, Lisowski V, Martinez J, Khrestchatisky M (2010) Synthetic therapeutic peptides: science and market. Drug discovery today 15(1): $40-56$

3. AM Thayer (2011) Improving peptides, Chemical \& Engineering News Archive. 89(22): 13-20.

4. Sato AK, Viswanathan M, Kent RB, Wood CR (2006) Therapeutic peptides: technological advances driving peptides into development. Current opinion in biotechnology 17(6): 638-642.

5. J Thundimadathil (2012) Cancer Treatment Using Peptides: Current Therapies and Future Prospects. Journal of Amino Acids p. 13.

6. Borghouts C, Kunz C, Groner B (2005) Current strategies for the development of peptide-based anti-cancer therapeutics. Journal of Peptide Science 11(11): 713-726.

7. Reichert J, Pechon P, Tartar A, Dunn M (2010) Development trends for peptide therapeutics, San Diego, CA: Peptide Therapeutics Foundation (PTF).

8. DP Mc Gregor (2008) Discovering and improving novel peptide therapeutics. Current opinion in pharmacology 8(5): 616-619.

9. Ladner RC, AK Sato, Gorzelany J, M De Souza (2004) Phage displayderived peptides as therapeutic alternatives to antibodies. Drug discovery today 9(12): 525-529.

10. Z Antošová, M Macková, V Král, T Macek (2009) Therapeutic application of peptides and proteins: parenteral forever? Trends in biotechnology 27(11): 628-635

11. Pichereau C, Allary C (2005) Therapeutic peptides under the spotlight. Eur Biopharm 5: 88-91. 
12. Langer R, Lund D, Leong K, Folkman J (1985) Controlled release of macromolecules: Biological studies. Journal of Controlled Release 2: 331-341.

13. MH Van Regenmorte (2001) Antigenicity and immunogenicity of synthetic peptides. Biologicals : journal of the International Association of Biological Standardization 29(3): 209-213.

14. BL Bray (2003) Large-scale manufacture of peptide therapeutics by chemical synthesis. Nature reviews. Drug discovery 2(7): 587-593.

15. Singh R, Singh S, Lillard JW (2008) Past, present, and future technologies for oral delivery of therapeutic proteins. Journal of pharmaceutical sciences 97(7): 2497-2523.

16. Werle M, Bernkop Schnürch A (2006) Strategies to improve plasma half life time of peptide and protein drugs. Amino Acids 30(4): 351-367.

17. Hamamoto K, Kida Y, Zhang Y, Shimizu T, Kuwano K (2002) Antimicrobial Activity and Stability to Proteolysis of Small Linear Cationic Peptides with D-Amino Acid Substitutions. Microbiology and Immunology 46(11): 741-749.

18. V Böttger, SF Howard, SM Picksley, P Chène, C Garcia Echeverria, et al. (1996) Identification of novel mdm2 binding peptides by phage display. Oncogene 13(10): 2141-2147.

19. W Eldridge, K Fitzgerald, N Cooley, D Mc Gregor (2006) Peptide stabilizer compounds and screening methods. Patent Application W02006097748.

20. PK Tsai, D Volkin, J Dabora, K Thompson, M Bruner, et al. (1993) Formulation Design of Acidic Fibroblast Growth Factor. Pharm Res 10(5): 649-659.

21. OL Johnson, JL Cleland, HJ Lee, M Charnis, E Duenas, et al. (1996) A month-long effect from a single injection of microencapsulated human growth hormone. Nature medicine 2: 795-799.

22. NB Bam, TW Randolph, JL (1995) Cleland Stability of Protein Formulations: Investigation of Surfactant Effects by a Novel EPR Spectroscopic Technique. Pharm Res 12(1): 2-11.

23. M Diago, J Crespo, A Olveira, R PÉRez, R BÁRcena, et al. (2007) Clinical trial: pharmacodynamics and pharmacokinetics of re-treatment with fixed-dose induction of peginterferon $\alpha-2 \mathrm{a}$ in hepatitis $\mathrm{C}$ virus genotype 1 true non-responder patients. Alimentary Pharmacology \& Therapeutics 26(8): 1131-1138.

ISSN: 2574-1241

DOI: 10.26717/BJSTR.2018.08.001694

Yusuf A Haggaga. Biomed J Sci \& Tech Res

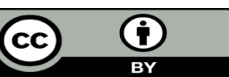

This work is licensed under Creative Commons Attribution 4.0 License

Submission Link: https://biomedres.us/submit-manuscript.php
24. T Doan, E Massarotti (2005) Rheumatoid Arthritis: An Overview of New and Emerging Therapies. The Journal of Clinical Pharmacology 45(7): 751-762.

25. V Balan, DR Nelson, MS Sulkowski, GT Everson, LR Lambiase, et al. (2006) A Phase I/II study evaluating escalating doses of recombinant human albumin-interferon-alpha fusion protein in chronic hepatitis C patients who have failed previous interferon-alpha-based therapy. Antiviral therapy 11(1): 35-45.

26. A Roisin, JP Robin, N Dereuddre Bosquet, AL Vitte, D Dormont, et al. (2004) Inhibition of HIV-1 replication by cell-penetrating peptides binding Rev. The Journal of biological chemistry 279: 9208-9214.

27. A Skerra (2000) Engineered protein scaffolds for molecular recognition. Journal of Molecular Recognition 13(4): 167-187.

28. A Muheem, F Shakeel, MA Jahangir, M Anwar, N Mallick, et al. (2016) A review on the strategies for oral delivery of proteins and peptides and their clinical perspectives. Saudi Pharmaceutical Journal: 24(4): 413428.

29. YA Haggag, AM Faheem (2015) Evaluation of nano spray drying as a method for drying and formulation of therapeutic peptides and proteins. Frontiers in Pharmacology 6: 140.

30. Y Haggag, Y Abdel Wahab, O Ojo, M Osman, S El Gizawy, et al. (2016) Preparation and in vivo evaluation of insulin-loaded biodegradable nanoparticles prepared from diblock copolymers of PLGA and PEG. International journal of pharmaceutics 499(1): 236-246.

31. YA Haggag, AM Faheem, MM Tambuwala, MA Osman, SA El Gizawy, et al. (2018) Effect of poly(ethylene glycol) content and formulation parameters on particulate properties and intraperitoneal delivery of insulin from PLGA nanoparticles prepared using the double-emulsion evaporation procedure. Pharmaceutical development and technology 23(4): 370-381.

32. YA Haggag, KB Matchett, H Dakir El, P Buchanan, MA Osman, et al. (2017) Nano-encapsulation of a novel anti-Ran-GTPase peptide for blockade of regulator of chromosome condensation 1 (RCC1) function in MDAMB-231 breast cancer cells. International journal of pharmaceutics 521(1): 40-53.

33. YA Haggag, MA Osman, SA El Gizawy, AE Goda, MM Shamloula, et al. (2018) Polymeric nano-encapsulation of 5-fluorouracil enhances anticancer activity and ameliorates side effects in solid Ehrlich Carcinomabearing mice. Biomedicine \& Pharmacotherapy 105: 215-224.

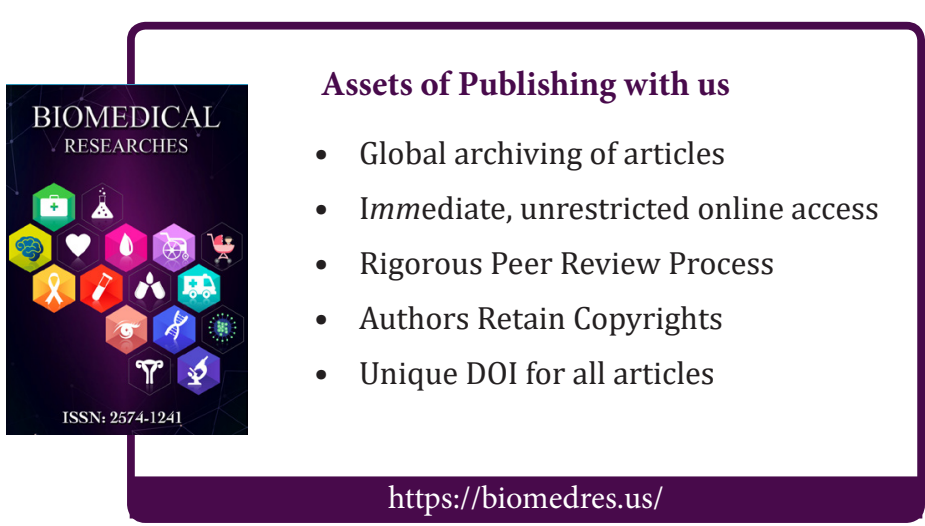

